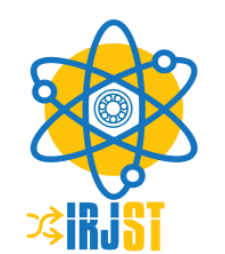

Available online at https://www.irjst.com/

International Research Journal of Science and Technology

ISSN: 2707-3955

DOI: https://doi.org/10.46378/irjst.2020.020102

\title{
First-Principle Study of Zinc Sulfide (Zinc Blende, Rock Salt and Wurtzite): Stability, Phase Transition and Structural Parameters
}

\author{
Ibrahim Isah ${ }^{1 *}$, Mustapha Isah ${ }^{2}$ \\ ${ }^{1}$ Department of Science Laboratory Technology, Jigawa State Polytechnic, Dutse, Jigawa State, Nigeria. \\ ${ }^{2}$ Department of Chemistry, Jigawa State College of Education, Gumel, Jigawa State, Nigeria.
}

\begin{tabular}{lll}
\multicolumn{2}{l}{ Paper Status } & \\
\hline Received & Nov 2020 \\
Accepted & $:$ & Nov 2020 \\
Published & $:$ & Dec 2020
\end{tabular}

Key Words

Phase Transition

Pseudopotential

Rock Salt

Semiconductors

Structural Parameters

Zinc Blende

Wurtzite

\section{Abstract}

The research investigates the stability, phase transition and structural parameters of zinc sulfide (Zinc blende, Rock salt and Wurtzite) using first-principle. The study employs generalized gradient approximation (GGA) within density functional theory (DFT) in which ultra-soft pseudopotential (Zn.pbe-van.UPF ${ }^{b}$ and S.pbe-van_bm.UPF ${ }^{c}$ ) were used for both zinc and sulfide respectively. Self-consistent calculation was made using cut-off energies of 26Ry $(\sim 350 \mathrm{eV})$ and $180 \mathrm{Ry}(\sim 2450 \mathrm{eV})$ for the cut-off wave function within the convergence accuracy of $\sim 1 \mathrm{mRy}$ with respect to total energy and $0.5 \mathrm{kbar}$ in case of pressure. The results obtained show that Wurtzite is more stable because it has lowest energy among the three structures, there is transition from zinc blende to rock salt and from Wurtzite to rock salt with transition pressures of $17.5 \mathrm{GPa}$ and $16.9 \mathrm{GPa}$ respectively and all the three polymorphs are semi-conductors due to their band gap.

Copyright () 2020: Ibrahim Isah, Mustapha Isah. This is an open-access distribution, and reproduction in any medium provided Access article distributed under the Creative Commons Attribution License the original work is properly cited License, which permits unrestricted use.

Citation: Ibrahim Isah, Mustapha Isah. "First-Principle Study of Zinc Sulfide (Zinc Blende, Rock Salt and Wurtzite): Stability, Phase Transition and Structural Parameters”. International Research Journal of Science and Technology, 2 (1), 313-318, 2020.

\section{Introduction}

Zinc sulfide $(\mathrm{ZnS})$ is one of the most important semiconductors and a subject of great interest in the recent years because of its technological importance and applications in science [1]. Its wide and direct band gap makes it ideal for applications in solar cells, laser diodes, high-density optical memory and electronic image display [1]. The electronic structure of a acompound plays a vital role in understanding the electrical, optical and transport properties of a material and helps in better interpretation of the solid state phenomena of that material [1].

Understanding the relationship between structural and electronic properties of the semiconductor materials and their phase transitions is important in designing their nanostructures with tunable properties [2].

\footnotetext{
* Corresponding Author: Ibrahim Isah

Department of Science Laboratory Technology, Jigawa State

Polytechnic, Dutse, Jigawa State, Nigeria

Email: abuabdallah99@gmail.com
}

Field pea (Pisum sativum) is one of the cool-season Zinc sulfide exists in three (3) polymorphs: cubic Zinc blende (B3), hexagonal Wurtzite (B4) and cubic rock salt (B1) which exist in rare. In the structure of B1 for $\mathrm{ZnS}$, the $\mathrm{Zn}$ and $\mathrm{S}$ atoms are at the positions: $(0,0,0)$ and $(1 / 2,1 / 2,1 / 2)$ respectively. For B3 structure of $\mathrm{ZnS}$, the $\mathrm{Zn}$ and $\mathrm{S}$ atom positions are $(0,0,0)$ and $(1 / 4,1 / 4,1 / 4)$ respectively. For B4 structure of $\mathrm{ZnS}$, there are four atoms per hexagonal unit cell where the positions of the atoms $\mathrm{Zn}$ and $\mathrm{S}$ are $(0,0,0),(1 / 3,2 / 3,1 / 2)$ and $(0,0, \mathrm{u})$, $(1 / 3,2 / 3, u+1 / 2)$, respectively, where $u$ is the dimensionless internal parameter that represents the distance between the $\mathrm{Zn}$ plane and its nearest neighbour $S$ plane. Below are the structures of the three Polymorphs of $\mathrm{ZnS}$ drawn using Xcrysden;

\section{Computational Method}

\subsection{Numerical Convergence}

The research work employs Generalized Gradient Approximation (GGA) within Density function theory (DFT). Ultra-Soft Pseudo potential (Zn.pbe-van.UPF ${ }^{\mathrm{b}}$ 
and S.pbe-van_bm.UPF ${ }^{c}$ ) were used for both Zinc and Sulfide respectively.

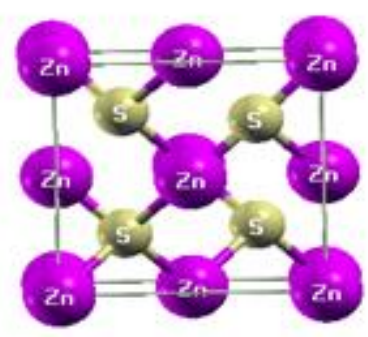

Fig. a. Zinc blend

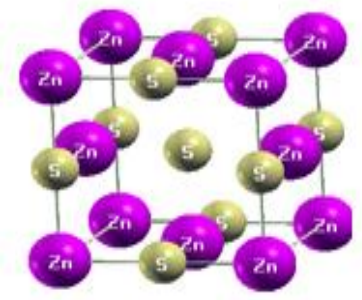

Fig. b. Rock salt

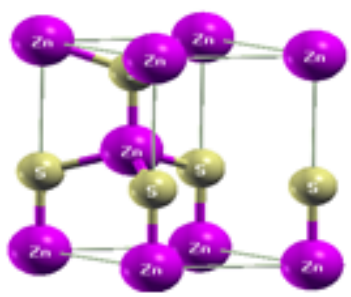

Fig. c. Wurtzite

A self-consistent calculation (scf) was made in which cut-off energies of 26Ry $(\sim 350 \mathrm{eV})$ and $180 \mathrm{Ry}$ $(\sim 2450 \mathrm{eV})$ were chosen for cut-off wave functions, they were chosen within convergence accuracy of $\sim 1 \mathrm{mRy}$ with respect to total energy and $0.5 \mathrm{kbar}$ in case of pressure [3]. The cut-offs were used for all the three structures B1, B3 and B4 (figure 1). This is done by setting the lattice parameters to the values obtained from the literatures [3-5].

The k-point was set to be $8 \times 8 \times 8$ initially in this case, later on the convergence with respect to k-points was also carried out in which the target convergence accuracy of $\sim 1 \mathrm{mRy}$ in total energy and $0.5 \mathrm{kbar}$ in pressure were obtained.

The k-point convergence was carried out next after setting the obtained convergence values for cut-off energies fixed. The convergence of k-point shown in figure 2, in which $6 \times 6 \times 6$ Monk horst-pack meshes was selected which give a convergence within the required accuracy of $\sim 1 \mathrm{mRy}$ energy/0.5kbar pressure.

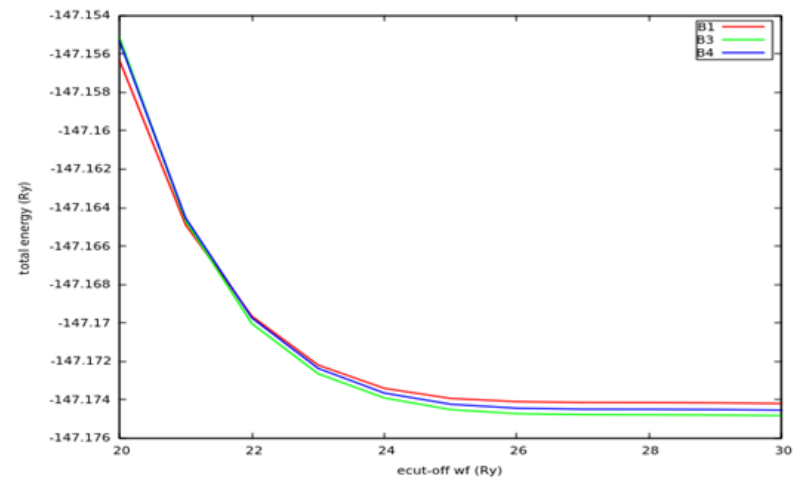

Fig.1a. Convergence of the total energy with respect to cut-off wave function.

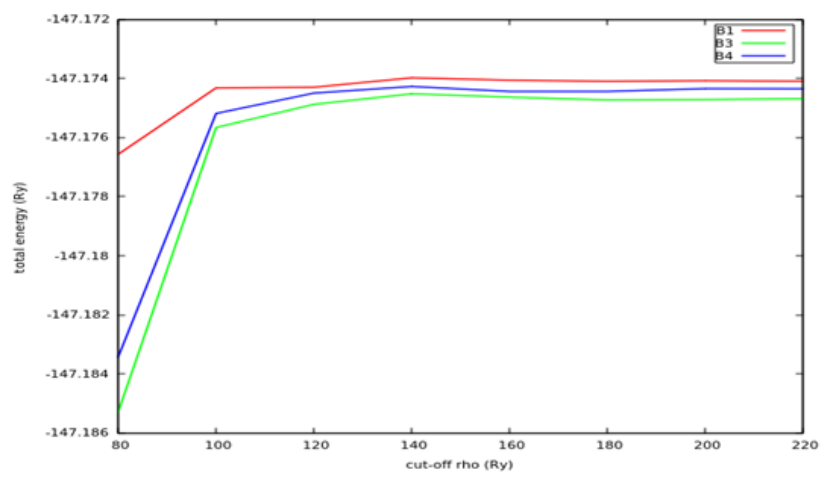

Fig.1b. Convergence of total energy with respect to cut-off rho

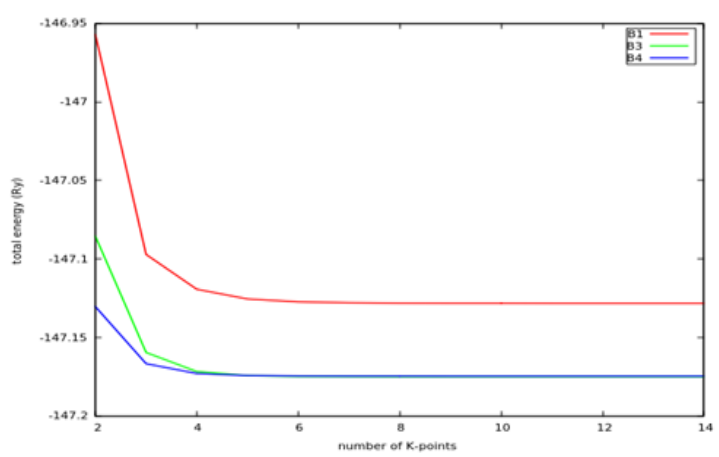

Fig.2a. Convergence of k-points with respect to total energy

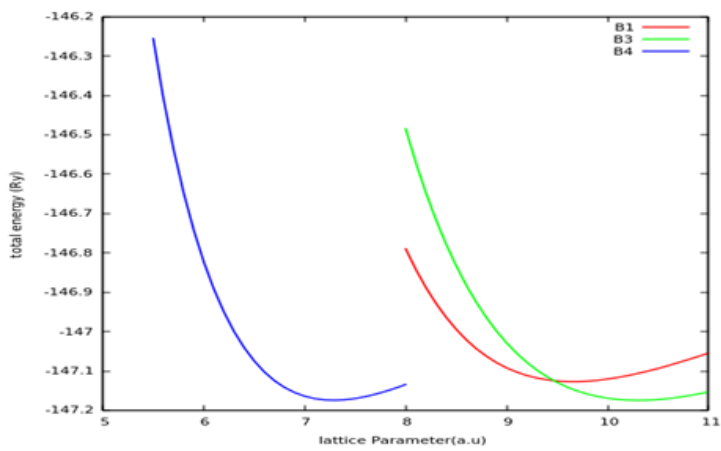

Fig.2b. Convergence of k-points with respect to pressure 
The three obtained convergence parameters, cut-off energies and k-points were fixed and the lattice parameters was then varied. An energy volume curve was plotted from the obtained data of the three structures (fig. 3). The transition phases were calculated from the energy-volume curve with two structures; Zinc blende and Rock salt (B3 to B1) and Wurtzite and Rock salt (B4 to B1). The phase behaviour, structural parameters, bulk modulus, pressure derivatives and equilibrium lattice parameters were obtained by fitting Birch-Murnaghan equation of the State [5].

The convergence test with respect to cut-off energies was repeated with the obtained k-points $6 \times 6 \times 6$ in which similar convergence was observed in both case; that is cut-off wave function and cut-off rho. Therefore, 6 k-points were selected for the remaining work throughout.

\subsection{Total Energy and Lattice Parameters}

The total energy was minimized in order to obtain the equilibrium lattice parameters for the Zinc blende, Rock salt and Wurtzite. The figure below (fig 3) shows the total energy versus lattice parameter curve for the three structures;

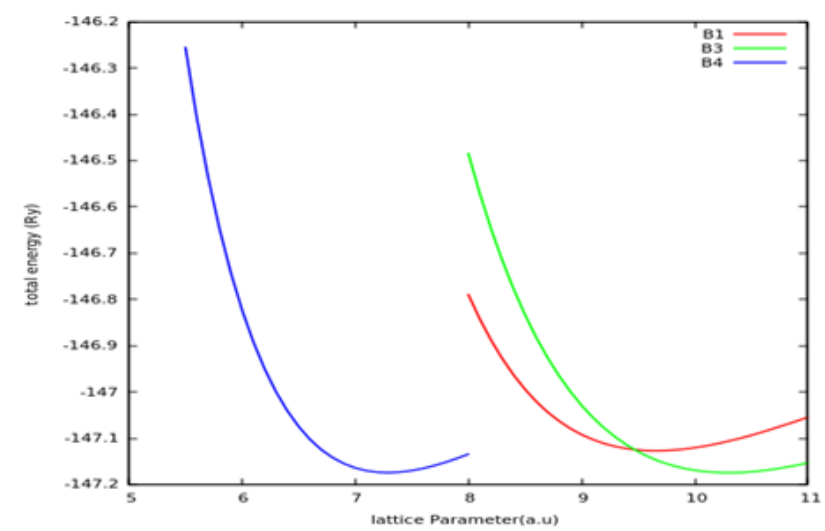

Fig.3. The lattice parameters for the three structures Zinc blende (B3), Rock salt (B1) and Wurtzite (B4)

\section{Results and Discussions}

The electronic properties of $\mathrm{ZnS}$ Polymorphs were presented in figures below (figure 4). The electronic properties of the ZnS Polymorphs, the band structures and the corresponding density of the state, for Zinc blende, Rock-salt and Wurtzite were presented below. The energy lattice parameters graphs were presented in figure 3 for Zinc blende (B3), Rock salt (B1) and Wurtzite (B4) in the same graph. The lattice parameters were obtained to be 10.3, 9.6 and 7.3 (a.u) for Zinc blende, Rock salt and Wurtzite respectively.

The ground-state properties of the rock-salt, Zinc blende and Wurtzite structures of $\mathrm{ZnS}$ were obtained by the minimization of the total energy, $\mathrm{E}$ (in Ry) with respect to the unit cell volumes, V, (in a.u ${ }^{3}$ ).
The energy-volume curve for Wurtzite (B4), Zinc blende (B3) and Rock salt (B1) structures were shown in the figure 5 below; As shown in the figure Wurtzite structure of the Zinc sulfide is more stable than the other two structures (Zinc blende and Rock salt) due to the fact that, the Wurtzite has lowest energy among the three structures.

In order to obtained the equilibrium lattice parameter of the three structures (Wurtzite, Zinc blende and Rock salt), Murnaghan's equation of state was used. The result is presented in Tables for the three structures B4, B3 and B1 respectively.

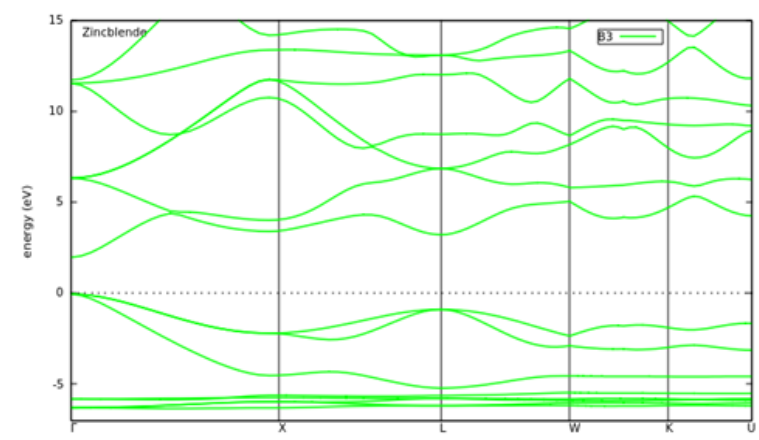

Fig.4a. Zinc blende Bands structure

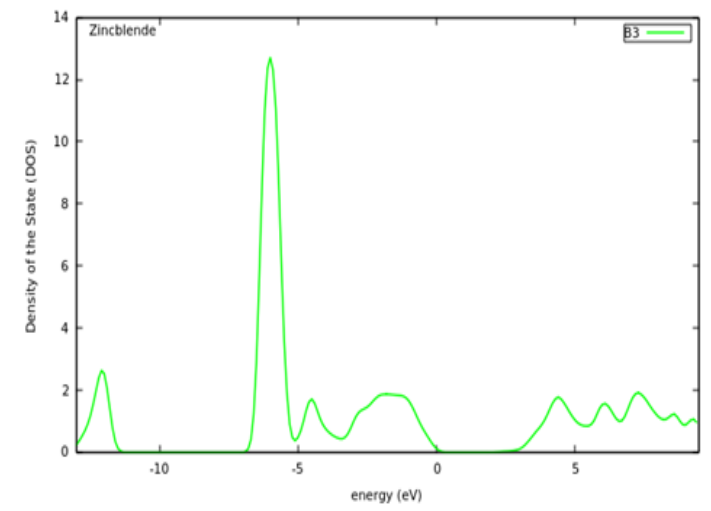

Fig.4b. Zinc blende Density of the state (DOS)

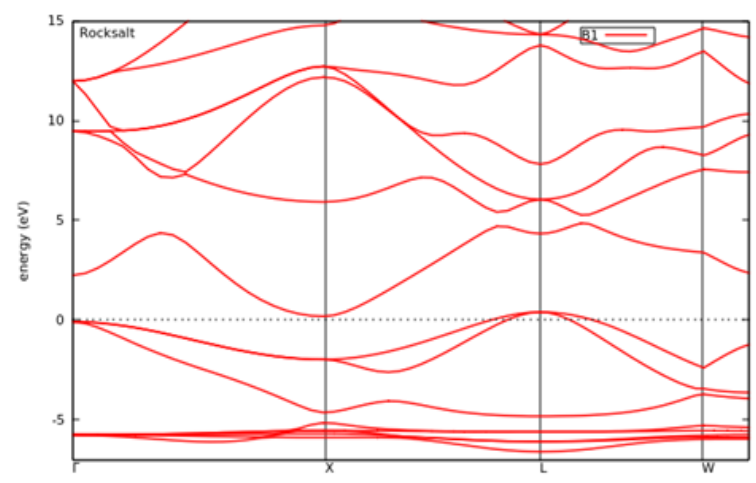

Fig.4c. Rock salt Band structure 


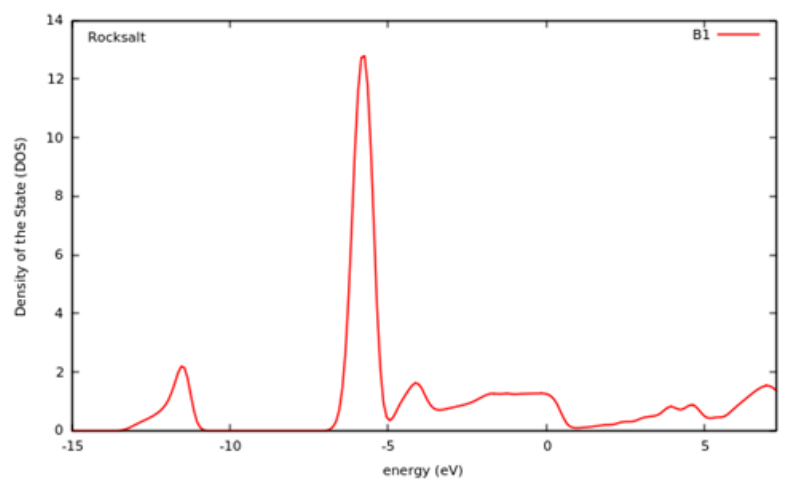

Fig.4d. Rock salt Density of the state (DOS)

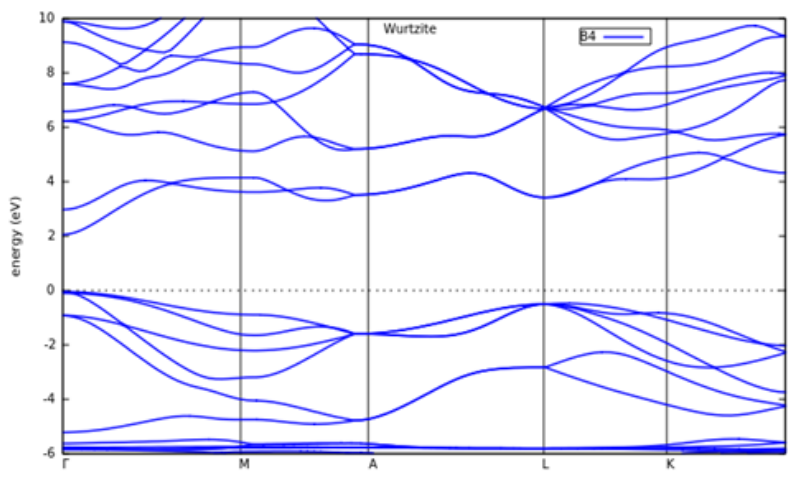

Fig.4e. Wurtzite Band structure

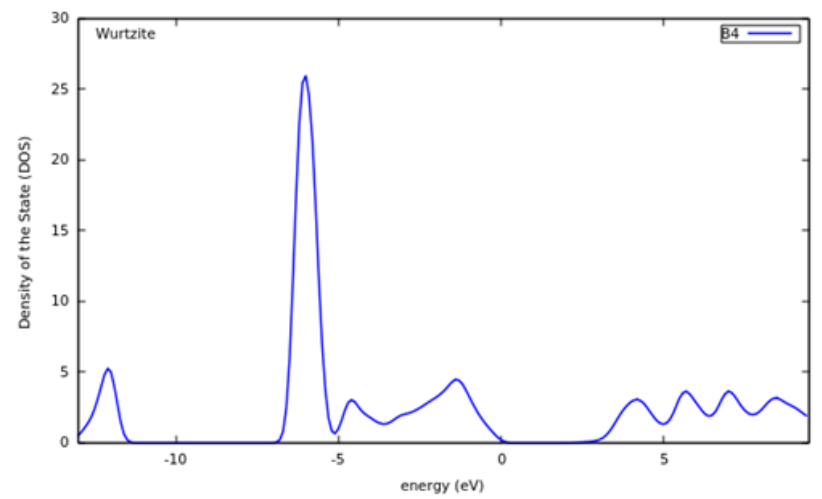

Fig.4f.Wurtzite Density of the state (DOS)

The Figures 4 ( $a, b, c, d$, e and f) shows that all the structures under study were semiconductors (Zinc blende, Rock salt and Wurtzite). This is due to their band gap.

Graph 6 (a and b) are for the transition pressure for Zinc blende to Rock salt for $6 \mathrm{a}$ which is found to be $17.5 \mathrm{GPa}$ and the transition pressure of Wurtzite to Rock salt $6 \mathrm{~b}$ which is found to be $16.9 \mathrm{GPa}$. Fig $6 \mathrm{c}$ is the enthalpy as function of pressure for Wurtzite and Zinc blende in which there is no transition.

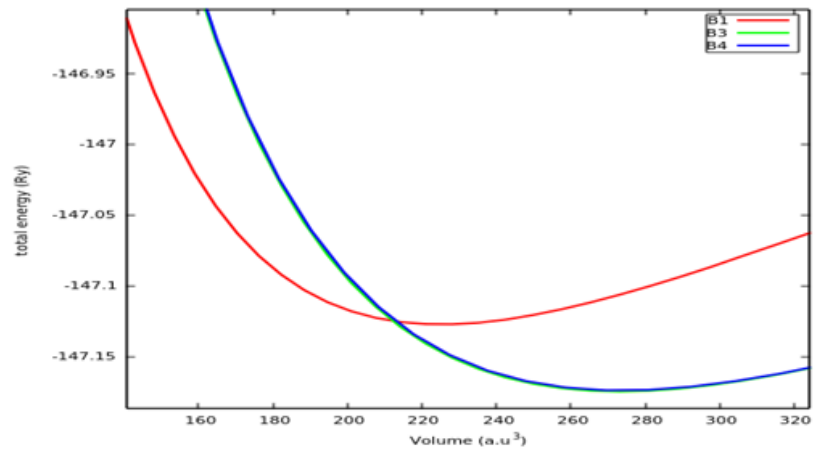

Fig.5.The energy-volume graph for the three structures (Rock salt(B1), Zinc blende(B3) and Wurtzite (B4)).

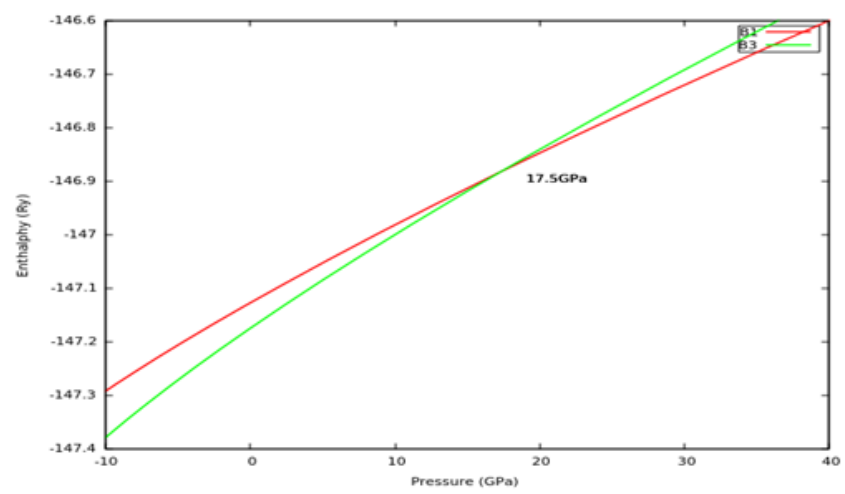

Fig.6a.Enthalpy as function of Pressure for the transition of Zinc blende to Rock salt

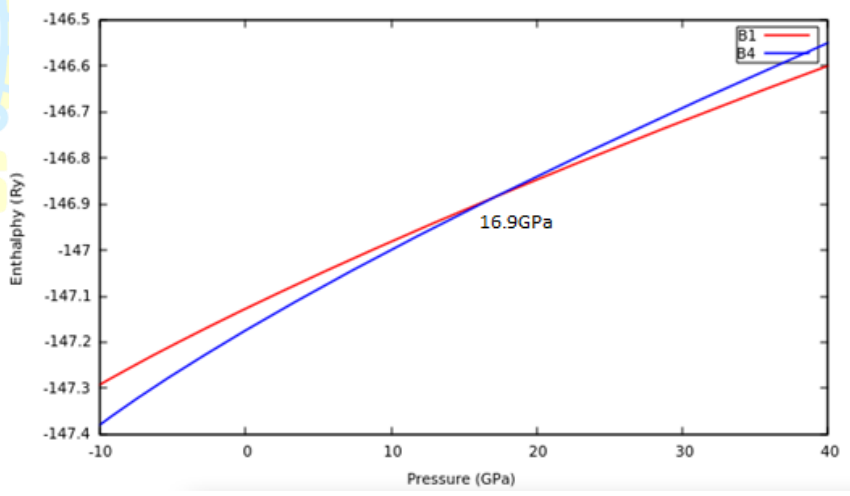

Fig.6b. Enthalpy as function of Pressure for the transition of Wurtzite to Rock salt

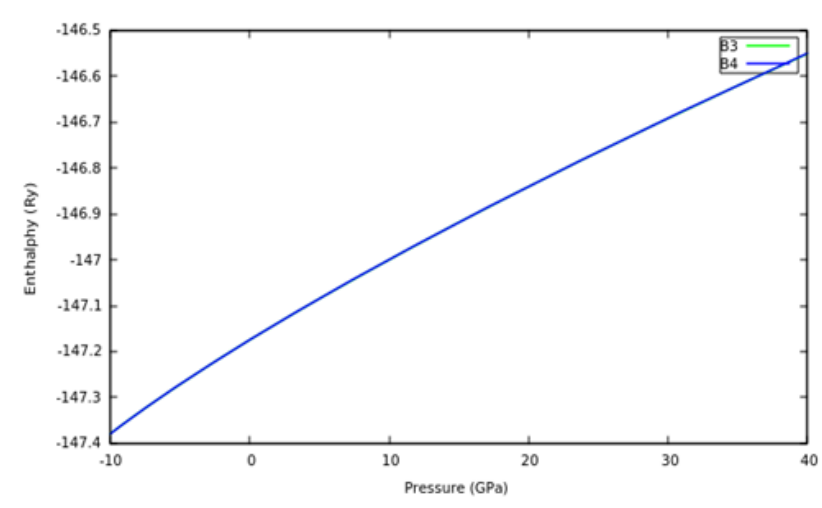

Fig.6c. Enthalpy as function of Pressure for the Wurtzite with Zinc blend 
Table1.Comparative Result between the StructuralParameters of the $\mathrm{ZnS}$ Polymorphs

\begin{tabular}{lllll}
\hline Polymorphs & $\mathrm{a}(\AA)$ & $\mathrm{c}(\AA)$ & $\mathrm{u}$ & Ref \\
\hline \multirow{4}{*}{ Zinc } & 5.44 & & 0.250 & \\
blende (B3) & 5.45 & - & 0.250 & {$[5]$} \\
& 5.42 & - & 0.250 & {$[2]$} \\
& 5.58 & - & 0.250 & {$[6]$} \\
\hline & 5.10 & - & 0.500 & \\
Rock & 5.11 & - & 0.500 & {$[5]$} \\
salt (B1) & 5.09 & - & 0.500 & {$[2]$} \\
& 5.31 & - & 0.500 & {$[6]$} \\
& $5.13-5.21$ & - & 0.500 & {$[7]$} \\
\hline & 3.85 & 6.30 & 0.375 & \\
Wurtzite & 3.85 & 6.30 & 0.375 & {$[5]$} \\
(B4) & 3.79 & 6.14 & 0.379 & {$[2]$} \\
& 3.811 & 6.234 & 0.375 & {$[8]$} \\
& 3.82 & 6.26 & 0.375 & {$[9]$} \\
\hline
\end{tabular}

Table 2. Comparative Results for Bulk Modulus, its Derivatives and Equilibrium Energies

\begin{tabular}{llll}
\hline Polymorphs & $\mathrm{B}_{0}(\mathrm{GPa})$ & $\mathrm{B}_{0}$ & Reference \\
\hline \multirow{2}{*}{ Zinc blende } & 75.7 & 3.43 & \\
& 71.75 & 4.19 & {$[3]$} \\
& 69.806 & 4.413 & {$[5]$} \\
\hline \multirow{3}{*}{ Rock salt } & 81.1 & 3.95 & \\
& 80.6 & 3.6 & {$[3]$} \\
& 85.107 & 4.510 & {$[5]$} \\
& $47.5-85.0$ & 4.0 & {$[7]$} \\
Wurtzite & 85.9 & 3.29 & \\
& 112.3 & 3.5 & \\
& 68.519 & 4.135 & {$[5]$} \\
\hline
\end{tabular}

The total energy versus volume of the three structures of $\mathrm{ZnS}$ from figure 5 show that, Zinc blende structure has less energy hence it is more stable among the others, even though there is a small energy difference between Zinc blende and Wurtzite. Hence, it was found that, theoretically most stable structure is the one that the material is found to prefer, that is Zinc blende

There is phase transition involved in the material, there is transition from Wurtzite to Rock salt and Zinc blende with transition pressures of $16.9 \mathrm{GPa}$ and $17.5 \mathrm{GPa}$ respectively (figure $6 \mathrm{~s}$ and $6 \mathrm{~b}$ ), it was reported to be $16 \mathrm{GPa}$ [10] and $18.1 \mathrm{GPa}$ [11] respectively. While in the case of figure $6 \mathrm{c}$ there is no transition between Zinc blende and Wurtzite.

\section{Conclusion}

In summary, first principle method based on the DFT (density function theory) was employed within GGA (generalized gradient approximation) to investigate the phase transitions, structural parameters and stability of ZnS structures (Zinc blende, Rock salt and Wurtzite).
It was found that there is phase transition from $\mathrm{B} 3$ to $\mathrm{B} 1$ and $\mathrm{B} 4$ to $\mathrm{B} 1$ with corresponding pressures of $16.9 \mathrm{GPa}$ and $17.5 \mathrm{GPa}$ respectively. It was also found that the material is most stable in its natural form, which is Zinc blende.

\section{References}

[1]. Kabita, K. \& Sharma, B. I. "First-principle study on structural, phase transition and electronic structure of Zinc Sulfide $(\mathrm{ZnS})$ within LDA, GGA and mBJ Potential", Journal of Physics: Conference Series 759, 2016.

[2]. La Porta, F. A., Gracia, L., Andres, J., Sambrano, J. R., Varela, J. A. \& Elson L. "A DFT study of Structural and Electronic Properties of $\mathrm{ZnS}$ Polymorphs and its Pressure-induced Phase Transition", Journal of American Ceramics Society, 97(12), PP. 1-8, 2014.

[3]. Badal, H. E. \& Nawzat, S. S. "First-principle Pseudopotential study of Zinc Blende to Rock Salt Phase Transition in ZnS", International Journal of Scientific and Engineering Research, 4(2), 2013.

[4]. Dong, M., Zhang, J. \& Yu, J. "Effect of effective mass and spontaneous polarization on photocatalytic activity of Wurtzite and Zinc blende ZnS", APL Materials, 3, PP. 1-9, 2015

[5]. Bilge, M., Ozdemir, K. S., Kart, H. H. \& Cagin, T. "Mechanical and electronic properties of $\mathrm{ZnS}$ under pressure", Journal of Achievements in Materials and Manufacturing Engineering, 31(1), PP. 29-34, 2008.

[6]. Jaffe, J. E., Pandey, R. \& Seel, M. J. “Ab initio high-pressure structural and electronic properties of ZnS", Journal of Physical Review B, 47(11), PP. 6299-6303, 1993.

[7]. Zhao, J. "First - principle study of atomic nitrogen solid with cubic gauche structure", Journal of Physics Letters A, 360, PP. 645648, 2007.

[8]. Xu, Y. \& Ching, W. Y. "Electronic, Optical and Structural properties of some Wurtzite crystals", Journal of Physical Review B, 48(7), PP. 4335-4351, 1993.

[9]. Kisi, E. H. \& Elcombe, M. M. "U Parameters for the Wurtzite structure of $\mathrm{ZnS}$ and $\mathrm{ZnO}$ using powder neutron diffraction", Acta Crystallographica, 45(12), PP.1867-1870, 1989. 
[10]. Hu, E. C., Sun, L. L., Zeng, Y. Z. \& Chen, R. $\mathrm{X}$. "Pressure-Temperature Induced Phase Transition of $\mathrm{ZnS}$ from First-Principles Calculations" Chinese Physics Letters, 25(2), PP. 675-678, 2008

[11]. Nazzal, A. \& Qteish, A. "Ab Initio Pseudopotential study of the structural phase transformations of $\mathrm{ZnS}$ under high Pressure", Journal of Physical Review B, 53(13), PP. 8262-8266, 1996. 